\title{
Viral capsids: Kinetics of assembly under transient conditions and kinetics of disassembly
}

\author{
Vladimir P. Zhdanov* \\ Section of Biological Physics, Department of Applied Physics, Chalmers University of Technology, S-41296 Göteborg, Sweden \\ and Boreskov Institute of Catalysis, Russian Academy of Sciences, Novosibirsk 630090, Russia
}

(Received 1 August 2014; published 27 October 2014)

\begin{abstract}
The available kinetic models of assembly of viral protein capsids are focused primarily on the situations in vitro where the amount of protein is fixed. In vivo, however, the viral protein synthesis and capsid assembly occur under transient conditions in parallel with viral genome replication. Herein, a kinetic model describing the latter case of capsid assembly is proposed with emphasis on the period corresponding to the initial stage of viral genome replication. The analysis is aimed at small icosahedral capsids. With biologically reasonable values of model parameters, the model predicts rapid exponential growth of the populations of monomers and fully assembled capsids during the transient period of genome replication. Under the subsequent steady-state conditions with respect to replication, the monomer population is predicted to be nearly constant while the number of fully assembled capsids increases linearly. The kinetics of capsid disassembly, described briefly as well under conditions of negligible monomer concentration, exhibit a short induction period when the number of proteins in a capsid is only slightly smaller than in the beginning, followed by more rapid protein detachment. According to calculations, the latter kinetics may strongly depend on protein degradation.
\end{abstract}

DOI: 10.1103/PhysRevE.90.042721

PACS number(s): 87.16.-b, 05.10.-a

\section{INTRODUCTION}

A viral DNA or RNA genome is protected by a protein capsid [1]. After penetration of a virion, V, through a hostcell lipid membrane, the capsid is removed and the genome is exposed to the intracellular environment. These steps are followed by genome $(\mathrm{G})$ replication,

$$
\mathrm{G} \rightarrow 2 \mathrm{G},
$$

mRNA (R) synthesis and degradation,

$$
\mathrm{G} \rightarrow \mathrm{G}+\mathrm{R} \text { and } \mathrm{R} \rightarrow \varnothing,
$$

protein $(\mathrm{P})$ synthesis and enzyme-mediated degradation,

$$
\mathrm{R} \rightarrow \mathrm{R}+\mathrm{P} \text { and } \mathrm{P} \rightarrow \varnothing,
$$

and formation of new virions,

$$
\mathrm{G}+m \mathrm{P} \rightarrow \mathrm{V}
$$

occurring either via G-mediated capsid assembly or via assembly of empty procapsids and capsids with subsequent incorporation of $\mathrm{G}$ ( $m$ is the number of proteins in a capsid). All these steps, shown here schematically on the coarse-grained level, occur typically via a few substeps, their specifics depend on the cell type and virus type, and the corresponding models and their analysis may be different.

The kinetic models describing various aspects of the viral replication cycle are now numerous (see, e.g., [2,3] and references therein). The kinetics of capsid assembly are typically not described in detail in such models. The available treatments of the kinetics of capsid assembly are focused on the case when the total amount of capsid protein is fixed (see, e.g., original studies [4-9], recent comprehensive review [10] and references therein; as a complementary material, see also a few recent articles concerning the capsid structure [11] and related molecular dynamics simulations [12]). This condition

\footnotetext{
*zhdanov@ chalmers.se
}

corresponds to the experiments in vitro. The situation when the concentration of monomers is fixed was briefly analyzed in [13]. The capsid assembly under steady-state conditions was also recently scrutinized [14]. In vivo, the assembly occurs in cells under transient conditions. The corresponding theoretical studies are just beginning. In particular, assembly of pentagonal building blocks into dodecahedral containers mediated by contacts with viral RNA was simulated in [15].

Herein, our main goal is to clarify theoretically the specifics of the kinetics of capsid assembly during the initial stage of viral genome replication. In addition, we also illustrate briefly the likely features of the disassembly kinetics. In vivo, the latter kinetics usually occur just after a virion entry into a cell. In vitro, such kinetics can be studied, e.g., by immobilizing virions at a membrane-covered surface (this platform is now widely used to explore various membrane processes [16]). Experiments of this type may shed light on the protein-protein interaction in capsids.

\section{CAPSID ASSEMBLY}

In our treatment, we use a minimal model focused on synthesis, attachment, detachment, and degradation of capsid proteins. In cells, these basic steps of capsid assembly occur in parallel with various related steps and depend on a multitude of factors, which are here either not described explicitly or neglected. For example, assembly of capsids often requires the assistance of scaffolding proteins [10] or may require the sequence-specific viral nucleic acid assistance [10] (e.g., in the case of single-stranded RNA viruses such as MS2 $[15,17])$. Packaging of viral genomes inside preassembled viral procapsids may be assisted by molecular motors [18]. In our analysis, scaffolding proteins are not described explicitly. Implicitly, however, the values of the attachment and detachment rate constants may depend on the concentration of scaffolding proteins. The effect of viral nucleic acid $[9,15,17]$ and/or molecular motors [18] is important and interesting but not always central in the kinetics of assembly. In the 
case of double-stranded DNA viruses and dsRNA viruses, for example, an appreciable part of capsids formed in cells is known to be often empty, i.e., does not contain genome [1] (b), and accordingly the sequence-specific assistance can be neglected. Here, the viral genome replication is not described explicitly and package of genome is not included either.

In our model, G replication (1) and mRNA formation (2) are considered to start at $t=0$. For capsid assembly, we use the scheme with monomer formation and degradation (3), conventional reversible attachment of monomers to $i$-mers (see, e.g., earlier models $[4-9,13]$ and general review of protein aggregation [19]),

$$
2 \mathrm{P} \rightleftharpoons \mathrm{P}_{2}, \quad \mathrm{P}+\mathrm{P}_{\mathrm{i}} \rightleftharpoons \mathrm{P}_{\mathrm{i}+1},
$$

and enzyme-mediated degradation of $i$-mers,

$$
\mathrm{P}_{i} \rightarrow \mathrm{P}_{i-1}
$$

where $2 \leqslant i \leqslant m-1$ (degradation of a fully assembled capsid is neglected; its inclusion does not change the results, as explained below). The assembly kinetics is described in terms of populations of monomers, $n_{1}(t)$, and $i$-mers, $n_{i}(t)$, with $2 \leqslant i \leqslant m$. At $t=0$, these populations are negligibly low, and we employ $n_{1}(0)=n_{i}(0)=0$ as the initial condition.

As already noticed in the Introduction, we are interested in the kinetics of capsid assembly during the initial stage of viral genome replication. Practically, this means that we analyze the situation when the genome replication is exponential including a short period after the exponential growth (at $0 \leqslant t \leqslant 2 \tau$ as defined below). During this stage, the release of newly formed virions from the cell is nearly negligible. Neglecting the latter process, we focus on steps (5) and (6).

With the specification above, the $\mathrm{P}$ population in the cell is described as

$$
d n_{1} / d t=w(t)-\kappa_{1} n_{1}-u
$$

where

$$
w(t)= \begin{cases}0 & \text { for } t<0, \\ w_{\circ}[\exp (t / \tau)-1] & \text { for } 0 \leqslant t \leqslant \tau, \\ w_{\circ}[\exp (1)-1] & \text { for } t>\tau,\end{cases}
$$

is the rate of $\mathrm{P}$ formation via mRNA translation (3), $\kappa_{1}$ is the rate constant of $\mathrm{P}$ degradation (3), and $u$ is the cumulative rate of the change of the P balance via steps (5) and (6). Using Eq. (8), we consider that the mRNA formation (2) starts at $t=0$ and then the mRNA population grows exponentially at $0 \leqslant t \leqslant \tau$ ( $\tau$ is the corresponding time scale) and is maintained constant at $t>\tau$ (the latter corresponds to steadystate conditions with respect to $\mathrm{G}$ replication and $\mathrm{mRNA}$ and $\mathrm{P}$ synthesis). In fact, we mimic the exponential mRNA growth. In principle, the $G$ replication and $m R N A$ and $P$ formation and degradation could be described explicitly [2,13], but this is beyond our present goals. One of the reasons is that, as already noticed, the details of $\mathrm{G}$ replication and mRNA formation depend on the cell type and virus type. Such details are still not complete, the corresponding parameter are numerous, and their validation is far from straightforward. The use of Eq. (8) allows us to avoid these complications, to focus on capsid assembly (or disassembly), and to keep the model generic.
For the populations of $i$-mers with $2 \leqslant i \leqslant m-2$, the kinetic equations describing steps (5) and (6) are read as

$$
\begin{aligned}
d n_{i} / d t= & k_{i-1} n_{i-1} n_{1} / V-k_{i} n_{i} n_{1} / V \\
& -r_{i} n_{i}+r_{i+1} n_{i+1}-\kappa_{i} n_{i}+\kappa_{i+1} n_{i+1},
\end{aligned}
$$

where $k_{i}$ and $r_{i}$ are the $\mathrm{P}$ attachment and detachment rate constants $\left(k_{i}\right.$ is calculated in $\left.\mathrm{cm}^{3} / \mathrm{min}\right), n_{1} / V$ is the intracellular $\mathrm{P}$ concentration ( $V$ is the cell volume), and $\kappa_{i}$ is the rate constant of degradation (6). The equations for the populations of $i$-mers with $i=m-1$ and $i=m$ are, respectively, represented as

$$
\begin{gathered}
d n_{i} / d t=k_{i-1} n_{i-1} n_{1} / V-k_{i} n_{i} n_{1} / V-r_{i} n_{i}-\kappa_{i} n_{i}, \\
d n_{i} / d t=k_{i-1} n_{i-1} n_{1} / V-r_{i} n_{i} .
\end{gathered}
$$

According to these equations, the cumulative rate of the change of the P balance via steps (5) and (6) is given by

$$
\begin{aligned}
u= & 2 k_{1} n_{1}^{2} / V-2 r_{2} n_{2}-\kappa_{2} n_{2}-r_{m} n_{m} \\
& +\sum_{i=3}^{m-1}\left(k_{i} n_{i} n_{1} / V-r_{i} n_{i}\right) .
\end{aligned}
$$

In Eq. (11) for fully assembled capsids, we keep the term describing protein detachment. In reality, such capsids often undergo a maturation reaction that involves covalent modification and/or conformational rearrangements enhancing the capsid stability [10]. Our model does not include these steps because we describe capsid assembly in a cell in the situation during and just after the initial transient G-replication period (a few hours). In the end of this period, the $\mathrm{P}$ population becomes large and the $\mathrm{P}$ detachment from assembled capsids is anyway eventually nearly negligible because it is fully compensated by attachment. (The situation with protein degradation in completely assembled capsids is similar.)

The rate constants $k_{i}, r_{i}$, and $\kappa_{i}$ depend on $i$. The corresponding expressions are determined by capsid symmetry. We are interested in icosahedral (nearly spherical) capsids (this is a feature of roughly half the virus families $[1,10])$. In this case, a partially formed capsid (with $i<m$ ) can be viewed as a truncated sphere with a circular rim for $P$ attachment [5]. The number of proteins located at the rim can be identified with the rim length measured in the monomer size, $a$ (by definition, this size and the capsid radius, $R$, are related as $4 \pi R^{2}=m a^{2}$ ). This length is represented as [5]

$$
l(i)=2 \pi^{1 / 2}[i(1-i / m)]^{1 / 2} .
$$

This approximate expression can be used for $2 \leqslant i \leqslant m-1$.

In our model, the $\mathrm{P}$ attachment is considered to be proportional to $l(i)$. Taking into account that biochemical reaction of association usually occur with low activation energy, we neglect the dependence of the $\mathrm{P}$ attachment activation energy on $i$. With these two approximations, the attachment rate constants are represented as

$$
k_{i}= \begin{cases}k_{\circ} & \text { for } i=1, \\ l(i) k_{\circ} & \text { for } 2 \leqslant i \leqslant m-1,\end{cases}
$$

where $k_{\circ}$ is the rate constant corresponding to $i=1$.

The $\mathrm{P}$ detachment may occur from the close-packed area and from the rim, and accordingly $r_{i}$ can be represented as a 
sum of the rate constants corresponding to these channels,

$$
r_{i}=r_{1, i}+r_{2, i} .
$$

The activation energy for the former channel is higher than that for the latter channel. If on the other hand $i$ is close to $m$, the number of monomers which may detach from the close-packed area is appreciably larger than the number of monomers forming the rim. For these reasons, the former channel is usually negligible during capsid assembly but may be significant provided $i \simeq m$ (e.g., in the very beginning of capsid disassembly). In the latter limit, almost every monomer in a procapsid is located in the close-packed area, and the rate constants for this channel are proportional to $i$, i.e.,

$$
r_{1, i}=i r_{\circ} \exp \left(-\mathcal{E} / k_{\mathrm{B}} T\right),
$$

where $r_{\circ}$ is the constant playing the role of a pre-exponential factor, and $\mathcal{E}$ is the activation energy. If $i$ is small, this expression is inaccurate. In the latter limit, the contribution of $r_{1, i}$ to $r_{i}$ is, however, anyway low. Thus, expression (16) can formally be used down to $i=2$.

During capsid assembly, the $\mathrm{P}$ detachment occurs typically at the rim [the second term in Eq. (15)]. The rate constants of the latter channel are here considered to be proportional to $l(i)$. In addition, we take the effect of the rim energy, $\gamma l(i)(\gamma$ is the line tension), on the activation energy of detachment via this channel. The change of the rim energy during detachment, $\gamma[l(i)-l(i-1)]$, is assumed to determine the dependence of the detachment activation energy on $i$. Physically, this approximation is similar to that used in the Kelvin model for evaporation of atoms from metal nanoparticles [20]. Following this line, we have

$$
r_{2, i}=\left\{\begin{array}{cl}
l(i) r_{\circ} \exp \left(-E_{i} / k_{\mathrm{B}} T\right) & \text { for } 2 \leqslant i \leqslant m-1, \\
0 & \text { for } i=m,
\end{array}\right.
$$

with

$$
E_{i}=E_{\circ}+\gamma[l(i-1)-l(i)],
$$

where $E_{\circ}$ is the activation energy in the limit of negligible rim curvature. A priori, one could expect that expression (18) would be valid only provided the difference $i-2$ or $m-i$ is appreciable. In fact, however, the term $\gamma l(i)$ describes fairly accurately the dependence of the capsid free energy on $i$ even if $i$ is close to 2 or $m[5,6]$. This validates the use of Eq. (18) for $2 \leqslant i \leqslant m-1$.

Attachment of monomers to and detachment from the rim are reverse processes, and their rate constants, $k_{i}$ and $r_{2, i}$, should satisfy the detailed balance principle. One of the manifestation of this principle is that both rate constants are proportional to $l(i)$. Another manifestation following from the expressions for the free energies of monomers and $i$-mers (see, e.g., [5]) is that the corresponding pre-exponential factors should be related as

$$
k_{\circ}=r_{\circ} v,
$$

where $v$ is the volume comparable with that of a monomer.

Concerning the reversibility of attachment and detachment of monomers, we may add that introducing the $\mathrm{P}$ detachment from the close-packed area, we should in principle introduce also the corresponding attachment channel. The role of the latter channel is, however, typically minor during capsid assembly as well as during disassembly. For this reason, the latter channel is neglected in our calculations.

The enzyme-mediated protein degradation of $i$-mers is assumed to occur at the rim, and accordingly the corresponding rate constants are considered to be proportional to $l(i)$. These rate constants may in principle depend on the rim curvature (in analogy with the dependence of the rate constant of conversion of membrane proteins on the membrane curvature [21]). This effect is, however, not too important in the present context and accordingly neglected. What might be more important is the difference of degradation rate constants of monomers and $i$-mers with $i \geqslant 2$. Here, one might well expect that monomers would degrade faster than $i$-mers (with $i \geqslant 2$ ), since they are the most accessible to the cellular digestive machinery. To illustrate the role of this factor, we performed two series of calculations. First, the difference was neglected, and the degradation rate constants were represented as

$$
\kappa_{i}= \begin{cases}\kappa_{\circ} & \text { for } i=1, \\ l(i) \kappa_{\circ} & \text { for } 2 \leqslant i \leqslant m-1,\end{cases}
$$

where $\kappa_{\circ}$ is the rate constant corresponding to monomers. In the second series, degradation of monomers was took into account (with $\kappa_{1}=\kappa_{\circ}$ ), while degradation of $i$-mers with $i \geqslant 2$ was neglected $\left(\kappa_{i}=0\right)$.

\section{CAPSID DISASSEMBLY}

With minor modifications and suitable choice of initial conditions, the equations presented above can be used to describe capsid disassembly. Focusing on intracellular kinetics, we will analyze disassembly of a single capsid in the situation when there are no capsid protein in a cell. The process is assumed to be spontaneous (in vivo, it may sometimes occur via the cotranslational pathway [22]). In this case, the equations above can be rewritten in terms of the probabilities $p_{i}$ that the capsid retains $i$ proteins. Taking into account that after detachment the attachment of capsid proteins is practically negligible (because the capsid contains only $m$ proteins, and after detachment some of these proteins they will degrade rather than attach back), we neglect the attachment terms as well as the term describing the formation of monomers. With these simplifications, the equations for $p_{i}$ read as

$\frac{d p_{i}}{d t}= \begin{cases}-r_{i} p_{i} & (i=m), \\ r_{i+1} p_{i+1}-r_{i} p_{i}+\kappa_{i+1} p_{i+1}-\kappa_{i} p_{i} & (2 \leqslant i<m), \\ r_{i+1} p_{i+1}+\kappa_{i+1} p_{i+1}-\kappa_{i} p_{i} & (i=1) .\end{cases}$

The corresponding initial conditions are $p_{i}(0)=1$ for $i=m$ and $p_{i}(0)=0$ for $1 \leqslant i \leqslant m-1$.

\section{PARAMETERS}

The parameters we have are: $m, \tau, w_{\circ}, k_{\circ}, r_{\circ}, \epsilon$, and $\gamma$, and $\kappa_{\circ}$. The choice and validation of the values of these parameters, used in our calculations presented below, are as follows.

Bearing in mind small icosahedral viruses, we use $m=60$.

In eukaryotes, the duration of the exponential regime of $\mathrm{G}$ replication is two or three hours [1], and accordingly we set $\tau=200 \mathrm{~min}$. 
The rates of mRNA and protein synthesis are usually lower or about $1-10 \mathrm{~min}^{-1}$ (see, e.g., Sec. 3.6 in review [23]). The viral proteins are expected to be produced with comparable or higher rate, and we employ $w_{\circ}=30$ and $100 \mathrm{~min}^{-1}$.

In vitro, the biochemical reactions of association are often relatively rapid so that the corresponding rate constants are comparable to those predicted for the diffusion-limited case, i.e., the upper value of $k_{\circ}$ is $4 \pi D \rho$, where $D$ the protein diffusion coefficient, and $\rho$ is the length comparable to the protein size. Under such conditions, the rate constant of protein attachment to a partly assembled capsid can be somewhat smaller due to steric constrains. In vivo, protein diffusion is, however, slowed down due to macromolecular crowding. Thus, the difference between the diffusion- and kinetically limited association is expected to be minor. Implying the diffusion control, taking into account that inside cells the scale of the protein diffusion coefficient is $D \simeq 3 \times 10^{-6} \mathrm{~cm}^{2} / \mathrm{min}$ [23], and using $\rho=2 \times 10^{-7} \mathrm{~cm}$, we obtain $k_{\circ}=10^{-11} \mathrm{~cm}^{3} / \mathrm{min}$. Employing $V=10^{-8} \mathrm{~cm}^{3}$ as the cell volume, we have $k_{\circ} / V=$ $10^{-3} \min ^{-1}$.

Substituting $k_{\circ}=10^{-11} \mathrm{~cm}^{3} / \min$ and $v=3 \times 10^{-20} \mathrm{~cm}^{3}$ into Eq. (19), we have $r_{\circ}=3 \times 10^{8} \mathrm{~min}^{-1}$. For the corresponding activation energies, we employ the values, $\mathcal{E} / k_{\mathrm{B}} T=28$ and $E_{\circ} / k_{\mathrm{B}} T=22$, predicting capsid disassembly on the time scale of a few hours. In reality, the rate of capsid disassembly depends on various factors, and the whole process may be both faster (e.g., after the virion entry into a cell) and slower (e.g., after the escape of virions from a cell) than that in our calculations.

The line tension, $\gamma / k_{\mathrm{B}} T=4$, was chosen in agreement with [6].

The protein degradation rate constants are often in the range $0.01-0.1 \mathrm{~min}^{-1}$ [23], and accordingly we use $\kappa_{\circ}=$ $3 \times 10^{-2} \mathrm{~min}^{-1}$.

Due to capsid stabilization mentioned in the paragraph below Eq. (12), the values of the detachment and degradation rate constants for $i$ close to $m$ may during capsid disassembly be different compared to those during assembly. In our context, this factor is not important because our goal is to show qualitative features of assembly and disassembly kinetics rather than to compare in detail their time scales. For this reason, we use the same parameters for assembly and disassembly.

\section{RESULTS OF CALCULATIONS}

To illustrate and explain the model predictions, it is instructive first to compare the dependence of various rate constants on $i$. For protein attachment and degradation, the corresponding rate constants [Eqs. (14) and (20)] are considered to be proportional to $l(i)$. For detachment, one of the rate constants (16) is proportional to $i$, while another one (17) depends exponentially on $E_{i}$ (Fig. 1). As already noticed, the former rate constant becomes comparable with the latter one only at $i \rightarrow m$. The dependence of the total detachment rate constant on $i$ is much stronger than that for attachment (Fig. 2). For this reason, the detachment rate during capsid assembly is typically higher and lower than $k_{i} n_{1} / V$ for small and large $i$, respectively. Physically, this means that the assembly occurs via nucleation. The size of the critical nuclei

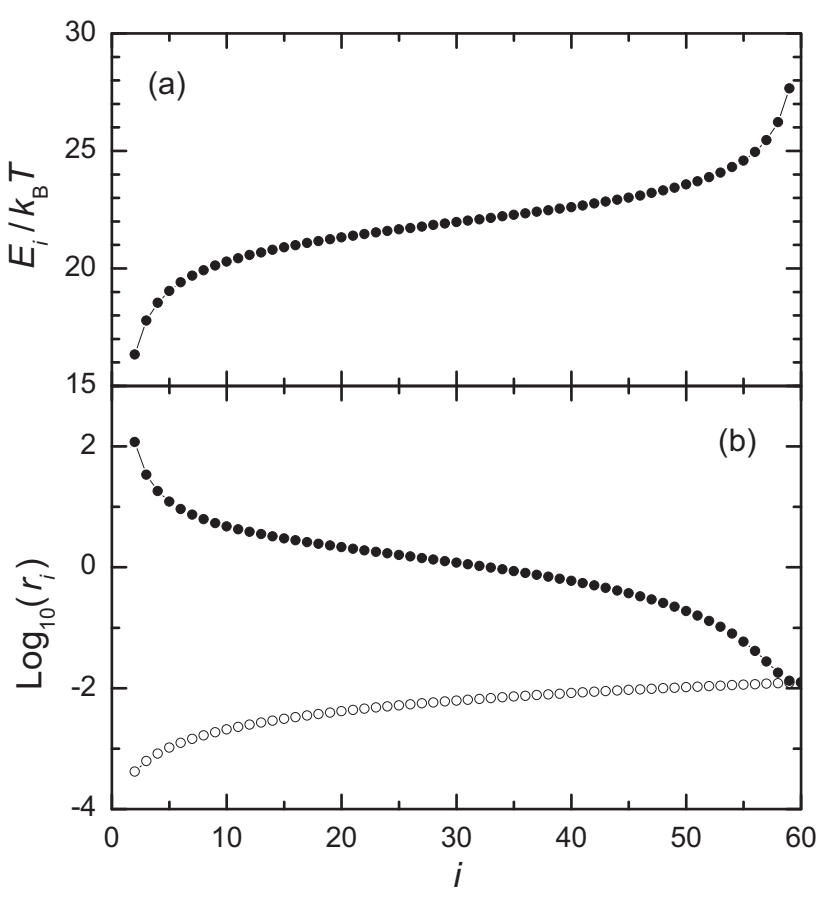

FIG. 1. Specification of the protein detachment rate: (a) the activation energy of detachment from the rim as a function of $i$; (b) the rate constants of detachment from the rim (filled circles) and close-packed area (open circles).

is determined by the condition $r_{i} \simeq k_{i} n_{1} / V$. The degradation rate is typically lower than the attachment rate but higher than the detachment rate provided $i$ is close to $m$.

Typical kinetics of capsid assembly calculated numerically (by using the standard Runge Kutta method) with and without (for $i \geqslant 2$ ) protein degradation are shown in Fig. 3 for $w_{\circ}=30$ and $100 \mathrm{~min}^{-1}$. During the first part of the transition period (at $0 \leqslant t \leqslant 100 \mathrm{~min}$ ), the monomer population is low, and the number of fully assembled capsids is negligible. During the second part of the transition period (at $100 \leqslant t \leqslant 200 \mathrm{~min}$ ), with increasing monomer population, the number of capsids

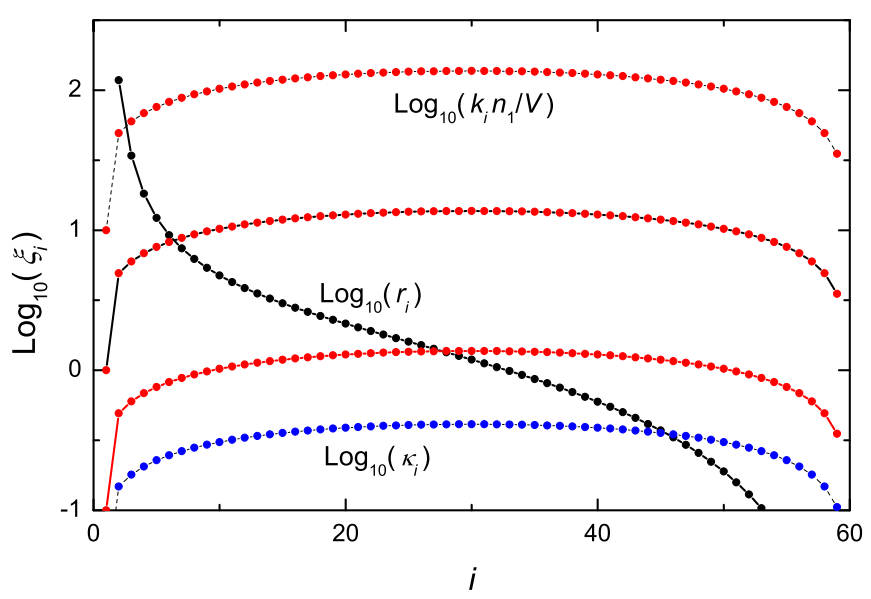

FIG. 2. (Color online) Specification of protein attachment, detachments and degradation rates: $\log _{10}\left(k_{i} n_{1} / V\right.$ ) (for $n_{1}=10^{2}, 10^{3}$, and $\left.10^{4}\right), \log _{10}\left(r_{i}\right)$, and $\log _{10}\left(\kappa_{i}\right)$ as a function of $i$. 


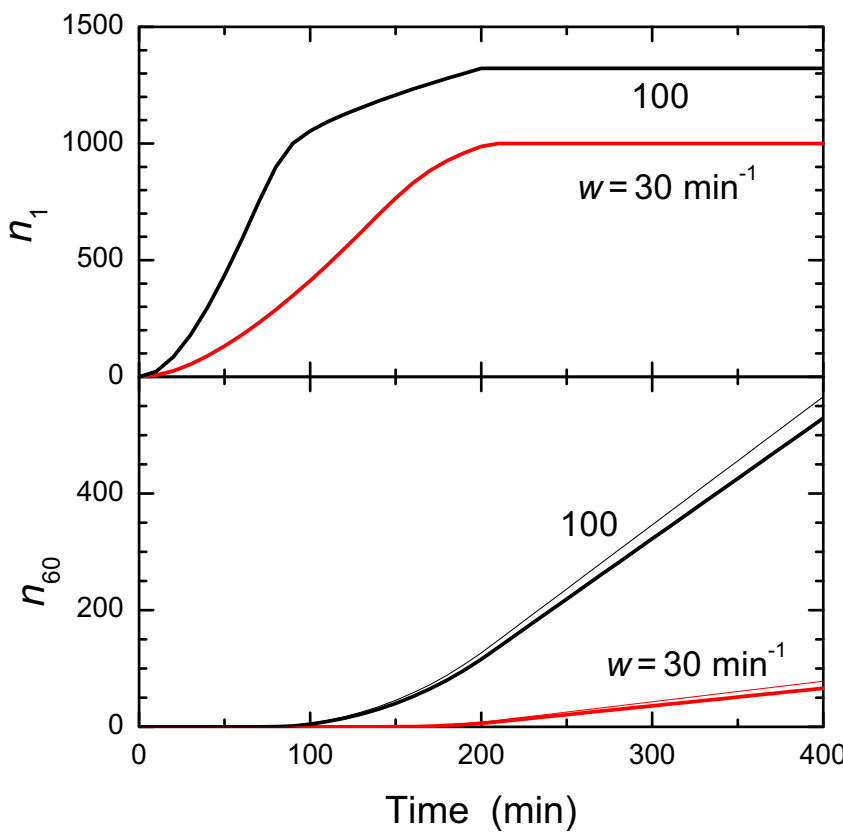

FIG. 3. (Color online) Numbers of monomers and fully assembled capsids as a function of time for $w_{\circ}=30$ and $100 \mathrm{~min}^{-1}$. The kinetics were calculated taking degradation of monomers into account. Degradation of $i$-mers (with $i \geqslant 2$ ) was either considered to occur (thick lines) or to be negligible (thin lines). In the case of monomers, the corresponding curves are not distinguishable by eye.

remains small if $w_{\circ}=30 \mathrm{~min}^{-1}$ but rapidly becomes appreciable if $w_{\circ}=100 \mathrm{~min}^{-1}$. Under steady-state conditions with respect to mRNA (at $t>200 \mathrm{~min}$ ), the monomer population is nearly constant while the number of capsids linearly increases. In all the cases (i.e., during transient and steady-state conditions), the number of partially assembled capsids is small compared to the number of monomers and fully assembled capsids as, e.g., shown in Fig. 4. The role of $i$-mer degradation (with $i \geqslant 2$ ) is minor. In particular, the kinetics with and without degradation are nearly identical.

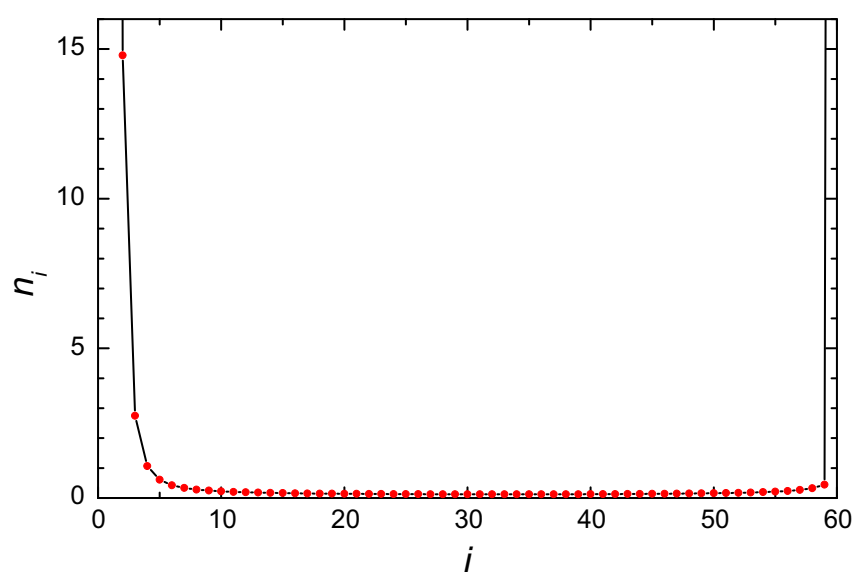

FIG. 4. (Color online) Distribution of dimers, trimers, and partially assembled capsids in the end of the kinetics calculated with $w_{\circ}=100 \mathrm{~min}^{-1}$ (thick lines in Fig. 3).

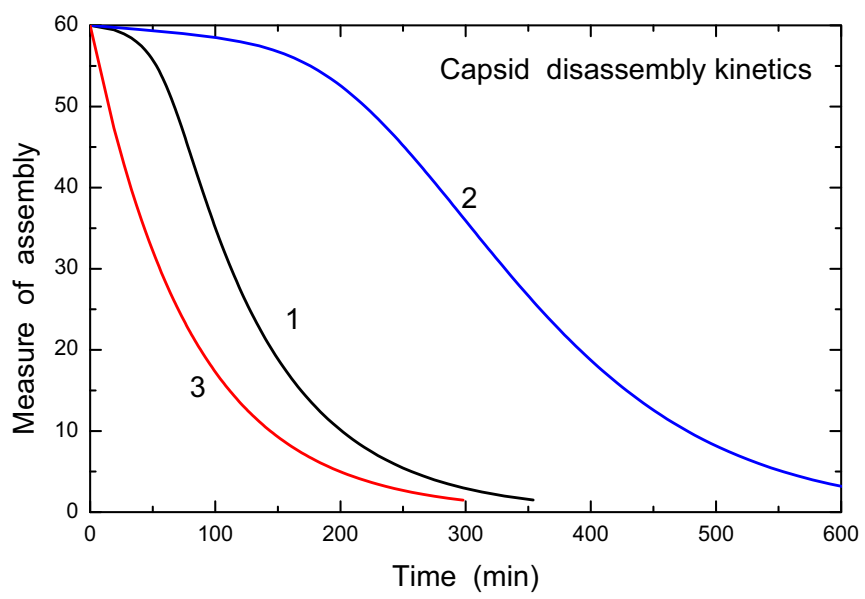

FIG. 5. (Color online) Kinetics of capsid disassembly with and without protein degradation. Lines 1 and 2 exhibit the average measure of capsid assembly, $\sum_{i=2}^{m} i p_{i}$, in these two cases. Line 3 shows $m p_{m}$. The latter value corresponding to the fully assembled capsid is the same in both cases.

Kinetics of capsid disassembly with and without protein degradation are exhibited in Fig. 5. In both case, there is relatively short induction period related to low protein detachment rate for $i$ close to $m$. In this limit, the rate of protein detachment is low, the protein degradation is important, and accordingly the kinetics with degradation is appreciably faster than that without degradation.

According to our calculations, the qualitative features of the kinetics under consideration are robust, i.e., can be observed in a wide range of parameters.

\section{CONCLUSION}

Taken together, the model and calculations presented help to understand the likely specifics of capsid assembly and disassembly kinetics in cells under transient conditions in a few hours after the beginning of viral genome replication. To articulate similarities and novelty of our results compared to those predicted earlier, it is instructive to outline the latter results and then compare them step by step. Such an outline, based on the in vitro measurements and theoretical treatments, was recently compiled in review [10] as follows: "(i) thermodynamically, the assembly reaction may be described as two-state: below a certain capsid proteins concentration, only the dissociated state (soluble capsid building blocks) is populated; above that concentration, only the fully associated state (complete capsids) is populated; few or no equilibrium intermediates are usually detected; (ii) the kinetics of capsid assembly follows a sigmoidal curve that includes a lag phase; (iii) the reaction rate is strongly dependent on protein concentration; the above observations together suggest that capsid assembly is a nucleation-and-growth process; (iv) at high protein concentrations, free capsid building blocks are depleted but only partially assembled capsids are formed, due to kinetic trapping; (v) there is hysteresis to dissociation: the capsid disassembles at much lower capsid protein concentrations than those required for assembly; (vi) depending on the conditions, off-pathway reactions may occur, leading to 
aberrant particles, capsids with non-native quaternary structure or polymorphisms."

Conclusions (i) and (iii) above are general (concerning (i), see, e.g., $[4,13,24]$ ) and fully applicable to our model. (ii) Our model predicts a lag phase followed eventually by linear increase of the number of complete capsids. This shape of the kinetics deviates from conventional sigmoidal curves which usually show a transition to constant population of complete capsids. In cells, the latter regime may be realized but to reproduce it, one is expected to introduce new steps, e.g., release of complete capsids from a cell. (iv) In our model, free capsid building blocks are depleted as well. (v) Hysteresis can be described in the framework of the model we use, but this was not our goal. Our analysis was rather focused on disassembly kinetics under condition of negligible population of monomers. In this case, the model predicts a short induction period when the number of capsid proteins is only slightly smaller than in the beginning, followed by more rapid protein detachment. The likely effect of protein degradation on the induction period is shown. (vi) This effect is not treated in our analysis.

In the in vivo-oriented studies, the kinetics of virion formation can be explored on the ensemble level or in individual cells. The interpretation of the former kinetics is far from straightforward [25] because they represent convolution of true kinetics each of which may depend on a few (often hidden) parameters. The measurements of kinetics of virion formation in individual cells is still challenge and such experiments are rare [25]. With this reservation, we may notice that the kinetics measured in single cells show (i) a lag phase followed by (ii) a linear phase and then by (iii) saturation [25]. The results of our calculations are in apparent qualitative agreement with observations (i) and (ii) [stage (iii) is not analyzed in our work].

Finally, we repeat and articulate that in vivo the viral assembly and disassembly may depend on various factors $[1,10]$. Our theoretical analysis was focused on the basic factors inherent to assembly and disassembly. Many aspects of the kinetics of viral assembly and disassembly in cells need and merit additional theoretical study.

\section{ACKNOWLEDGMENTS}

This work was supported by Swedish Research Council (Grant No. 2010-5063). The author thanks F. Höök for useful discussions.
[1] A. J. Cann, Principles of Molecular Virology (Elsevier, Amsterdam, 2012); Structure and Physics of Viruses, edited by M. G. Mateu (Springer, Heidelberg, 2013).

[2] J. Yin, AIChE J. 53, 2202 (2007); Y. Sidorenko, A. Voigt, J. Schulze-Horsel, U. Reichl, and A. Kienle, Chem. Eng. Sci. 63, 2299 (2008); J. Sardanye and S. F. Elena, PLoS ONE 6, e24884 (2011).

[3] M. Bally, K. Dimitrievski, G. Larson, V. P. Zhdanov, and F. Höök, Phys. Biol. 9, 026011 (2012); V. P. Zhdanov, Phys. Rev. E 88, 064701 (2013).

[4] D. Endres and A. Zlotnick, Biophys. J. 83, 1217 (2002).

[5] R. Zandi, P. van der Schoot, D. Reguera, W. Kege, and H. Reiss, Biophys. J. 90, 1939 (2006).

[6] A. Yu. Morozov, R. F. Bruinsma, and J. Rudnick, J. Chem. Phys. 131, 155101 (2009).

[7] M. F. Hagan and O. M. Elrad, Biophys. J. 98, 1065 (2010).

[8] E. C. Dykeman, P. G. Stockley, and R. Twarock, Phys. Rev. E 87, 022717 (2013).

[9] A. Zlotnick, J. Z. Porterfield, and J. C.-Y. Wang, Biophys. J. 104, 1595 (2013).

[10] M. G. Mateu, Arch. Biochem. Biophys. 531, 65 (2013).

[11] E. R. May and C. L. Brooks III, Phys. Rev. Lett. 106, 188101 (2011); A. L. Bozic, A. Siber, and R. Podgornik, J. Biol. Phys. 38, 657 (2012); S. Sirotkin, A. Mermet, M. Bergoin, V. Ward, and J. L. Van Etten, Phys. Rev. E 90, 022718 (2014); J. Kim and J. Wu, J. Chem. Phys. 140, 235101 (2014);
G. Erdemci-Tandogan, J. Wagner, P. van der Schoot, R. Podgornik, and R. Zandi, Phys. Rev. E 89, 032707 (2014).

[12] D. C. Rapaport, Phys. Biol. 7, 045001 (2010); Phys. Rev. E 86, 051917 (2012); E. R. May, Molec. Simul. 40, 878 (2014).

[13] V. P. Zhdanov, BioSystems 77, 143 (2004).

[14] M. Castelnovo, T. Verdier, and L. Foret, Europhys. Lett. 105, 28006 (2014).

[15] E. C. Dykeman, P. G. Stockley, and R. Twarock, Proc. Natl. Acad. Sci. USA 111, 5361 (2014).

[16] G. J. Hardy, R. Nayak, and S. Zauscher, Curr. Opin. Coll. Interf. Sci. 18, 448 (2013); T. G. Pomorski, T. Nylander, and M. Cardenas, Adv. Coll. Interf. Sci. 205, 207 (2014).

[17] E. C. Dykeman, P. G. Stockley, and R. Twarock, J. Mol. Biol. 425, 3235 (2013).

[18] N. Keller, D. delToro, S. Grimes, P. J. Jardine, and D. E. Smith, Phys. Rev. Lett. 112, 248101 (2014).

[19] A. M. Morris, M. A. Watzky, and R. G. Finke, Biochim. Biophys. Acta 1794, 375 (2009).

[20] S. C. Parker and C. T. Campbell, Phys. Rev. B 75, 035430 (2007).

[21] V. P. Zhdanov and F. Höök, Biophys. Chem. 170, 17 (2012).

[22] N. Christensen, J. Tilsner, K. Bell, P. Hammann, R. Parton, C. Lacomme, and K. Oparka, Traffic 10, 536 (2009).

[23] V. P. Zhdanov, Phys. Rep. 500, 1 (2011).

[24] R. Zandi and P. van der Schoot, Biophys. J. 96, 9 (2009).

[25] A. Timm and J. Yin, Virology 424, 11 (2012). 